\title{
PROTEIN-NUCLEIC ACID INTERFACE (PNAI) INHIBITOR DRUG MOLECULES FOR SARS-COV-2
}

\author{
Hamdullah Khadim Sheikh ${ }^{\mathrm{a}^{*}}$, Tanzila Arshad ${ }^{\mathrm{b}}$, Zainab Sher Mohammad $^{\mathrm{c}}$ Iqra Arshad $^{\mathrm{d}}$, \\ ${ }^{\mathrm{e}}$ Mohtasheemul Hassan
}

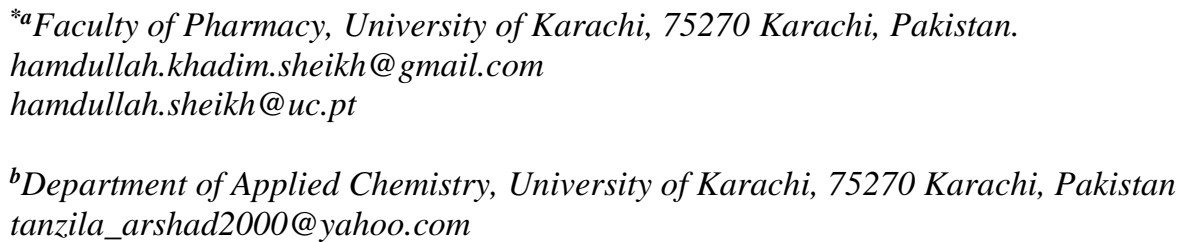

${ }^{c}$ Physical therapy, Health Sciences, Riphah International University, Islamabad, Pakistan zainabmohammad696@gmail.com

${ }^{d}$ Research Centre for Modelling \& Simulation, National University of Science \& Technology iarshad.msbi18rcms@nust.edu.pk

${ }^{e}$ Faculty of Pharmacy, University of Karachi, 75270 Karachi, Pakistan. mohassan@uok.edu.pk

1. Abstract

In this research we used the structure of SARS-CoV-2 related, recently mapped, atomic structure of nsp10/16 proteins for docking with some known drug molecular structures at $\mathrm{pH} 7$ and 5. Chosen molecules were azo $-\mathrm{N}=\mathrm{N}$ - and $-\mathrm{COOH}$ derivatives. It was revealed that the molecules showed good binding energy with nsp10/16 protein at both $\mathrm{pH}$. These molecules can act as proteinnucleic acid interface (PNAI) inhibitor drug molecules. Such molecules can be used in combination with polymerase and protease inhibitors for treatment of SARS-CoV-2.

Keywords: PNAI; Drug; SARS-CoV-2; Docking; NSP10/16

\section{Introduction}

SARS-COV-2 is a single positive strand RNA virus [1]. So far, the strategies to develop potent drugs against the SARSCOV-2 virus included inhibition of RNA dependent RNA polymerase (RdRp), angiotensin-converting enzyme II (ACE2) entry receptor and protease. Some of the identified RdRp molecular inhibitors targeting ACE2 have showed less specificity with side effects [2][3]. Protease (Mpro) proteins also act as drug targets for protease inhibitor molecules [4][5][6][7]. Recently, X-ray crystal structure of the SARS-COV-2 Mpro has been determined which provides an opportunity for structure-based protease inhibitor drug molecular designing [8]. Covalent binding protease inhibitors have shown side effects as well [9-14], while noncovalent protease inhibitors showed fewer side effects. Recent research work being done is focusing on known and clinically approved drug molecules [15-17]. In this research we used the same strategy of using known structures.

Recently Scientists from Northwestern University Feinberg School of Medicine have mapped the atomic structure of two critical proteins joined together in a complex, nsp10/16 [18]. The nsp10/16 protein is 
Docking of (PNAI) inhibitor molecules for SARS-COV-2

known as RNA methyltransferase or MTase. SARS-CoV-2 lacks replicase and transcriptase components. In order to replicate itself, the viral RNA reaches the cytoplasm of the human cell transcriptase and replicase components are synthesized as a result. These components include nonstructural viral proteins (nsp) which aid the virus to replicate. Hence, nsp proteins serve as potential SARS-CoV-2 target sites for anti-viral molecules. nsp10 plays a vital role in viral RNA synthesis where as nsp 16 aids in cap formation. Thus, to resist the viral replication, nsp10/16 should be inhibited by protein-nucleic acid interface (PNAI) inhibitors [19].

In this research, we used already known drug molecules so that other molecular and drug related properties are already known. This research can lead to further development of PNAI inhibitor drug molecules for binding with nsp10/16 proteins.

\section{Experimental}

Docking simulations were performed using MOE2015.10 software. Structures of all drug molecules were downloaded from PubChem website in .sdf format. By using Open Babel software [20] all structures were converted to. mol 2 format. For preparation of receptor, the structure of PDB $6 \mathrm{~W} 4 \mathrm{H}$ (resolution $1.80 \AA$ ) was obtained from the Protein Data Bank [21] in .pdb format. Docking simulations were performed using MOE2015.10 software at pH 7 and 5.

\section{Results and Discussion}

Docking analysis on nsp10/16 was done on the basis of hydrogen bonding, Van der Waals forces and $\pi$-stacking interactions. Binding energies of proposed protein-nucleic acid interactions (PNAI) inhibitors (1-10) are given in Table-1. Ligand and receptor interaction details are given in Table 2.1 and 2.2. Some of the selected molecules (1-2) are known PNAI inhibitors against SARS-COV Nsp15 [22]. Benzopurpurin B (1) has recently been tested on SARS-COV-2 infected patients [23]. (4-6) were selected based on their similar structure to the known PNAI inhibitor molecules (1-2). We also used aurintricarboxylic acid (ATA) (3) for docking with PNAI. This molecule was used in previous studies as selective inhibitor of nsp16 for SARS-COV [24].

The virus-infected cells produce ATP during oxidative metabolism and also by glycolysis. Glycolysis converts glucose $\left(\mathrm{C}_{6} \mathrm{H}_{12} \mathrm{O}_{6}\right)$ into pyruvate $\left(\mathrm{CH}_{3} \mathrm{COCOO}^{-}+\mathrm{H}^{+}\right)$which further converts into lactate. Because of high rates of glycolysis and synthesis of lactate, $\mathrm{pH}$ of the cell turns acidic [25]. In this research work, we checked the binding of the selected molecules (1-10) at both neutral and slightly acidic $\mathrm{pH}$.

Data from Table-1 demonstrates that all the selected protease inhibitors (1-10) bind with energy in between 5.6-10.1 -kcal/mol. At pH 5 , highest binding was showed by molecule (9) (Figure-1) while at $\mathrm{pH} \mathrm{7,} \mathrm{(5)} \mathrm{gave} \mathrm{the}$ highest binding energy (Figure-2). Almost all selected molecules showed least differential in the binding energies with changing $\mathrm{pH}$ except (5).

\section{Conclusion}

We used protein-nucleic acid interface (PNAI) as a receptor site for binding with selected $-\mathrm{N}=\mathrm{N}$ and $-\mathrm{COOH}$ based (PNAI) inhibitors (1-10) at $\mathrm{pH} 7$ and 5. It has been found that selected molecules showed fair level binding energies towards protein- 
Docking of (PNAI) inhibitor molecules for SARS-COV-2

nucleic acid interface (PNAI). The molecules we used for docking such as (1-3) are commercially available and have been proven effective in inhabiting replication of SARS-CoV. Hence, these molecules can lead to further development of proteinnucleic acid interface (PNAI) inhibitor drug molecules for SARS-CoV-2. Our group is working on further deep docking (DD)

Table 1: Binding energy evaluation data of molecules (1-10) with 6W4H (PNAI).

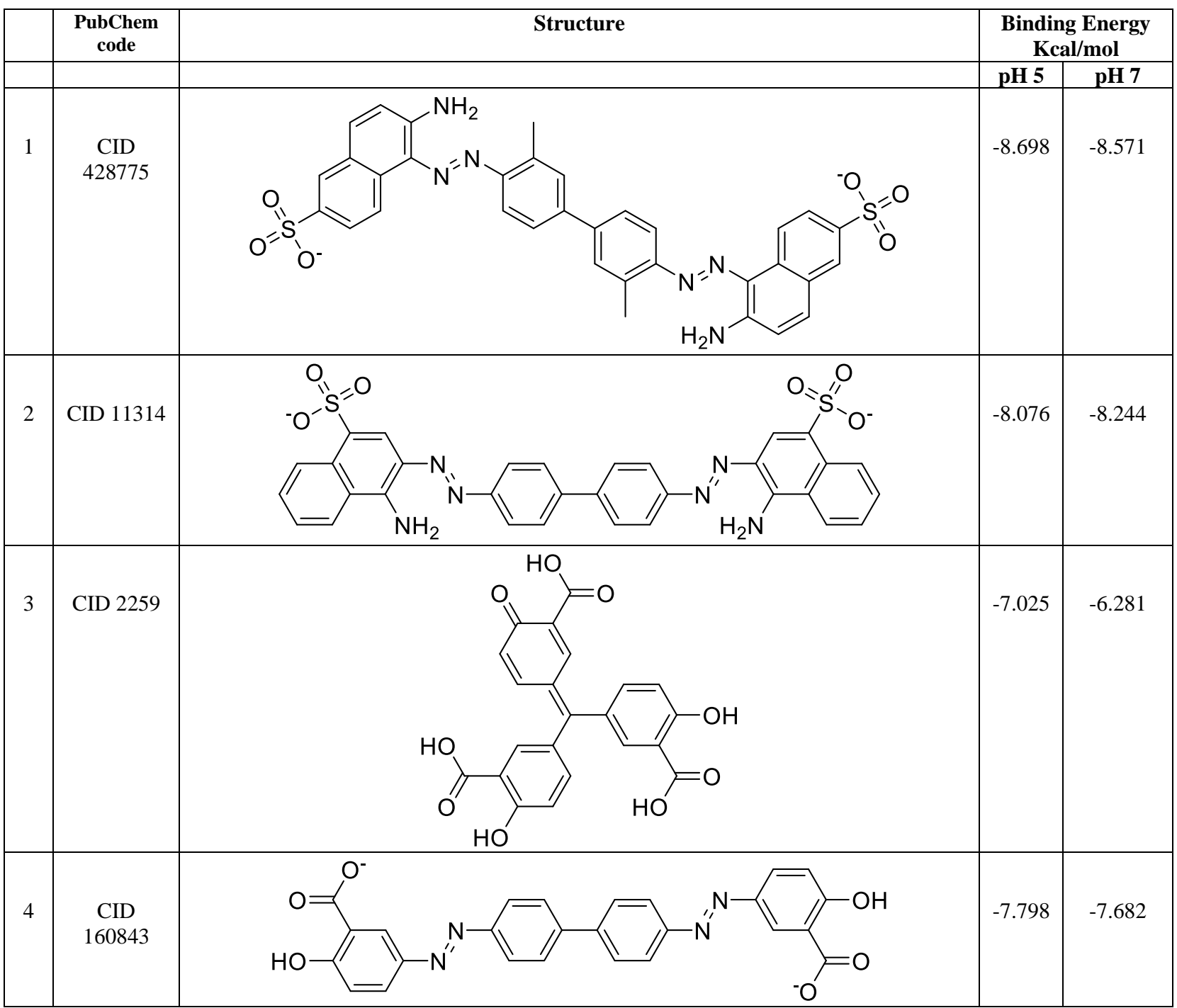

structural searches for more PNAI inhibitors for SARS-CoV-2 virus. PNAI inhibitors along with protease and polymerase inhibitors can be used to treat SARS-CoV-2.

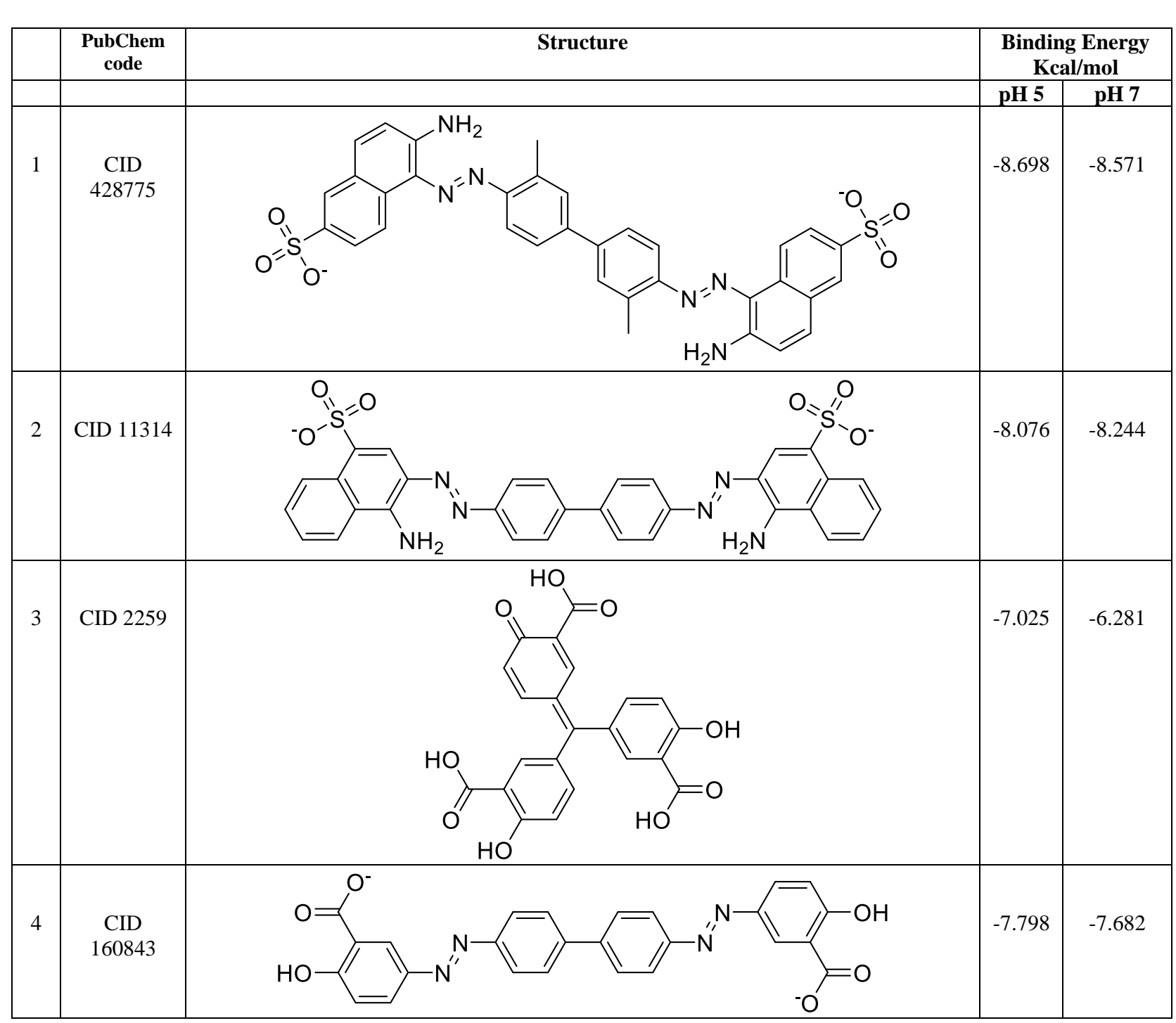


Docking of (PNAI) inhibitor molecules for SARS-COV-2

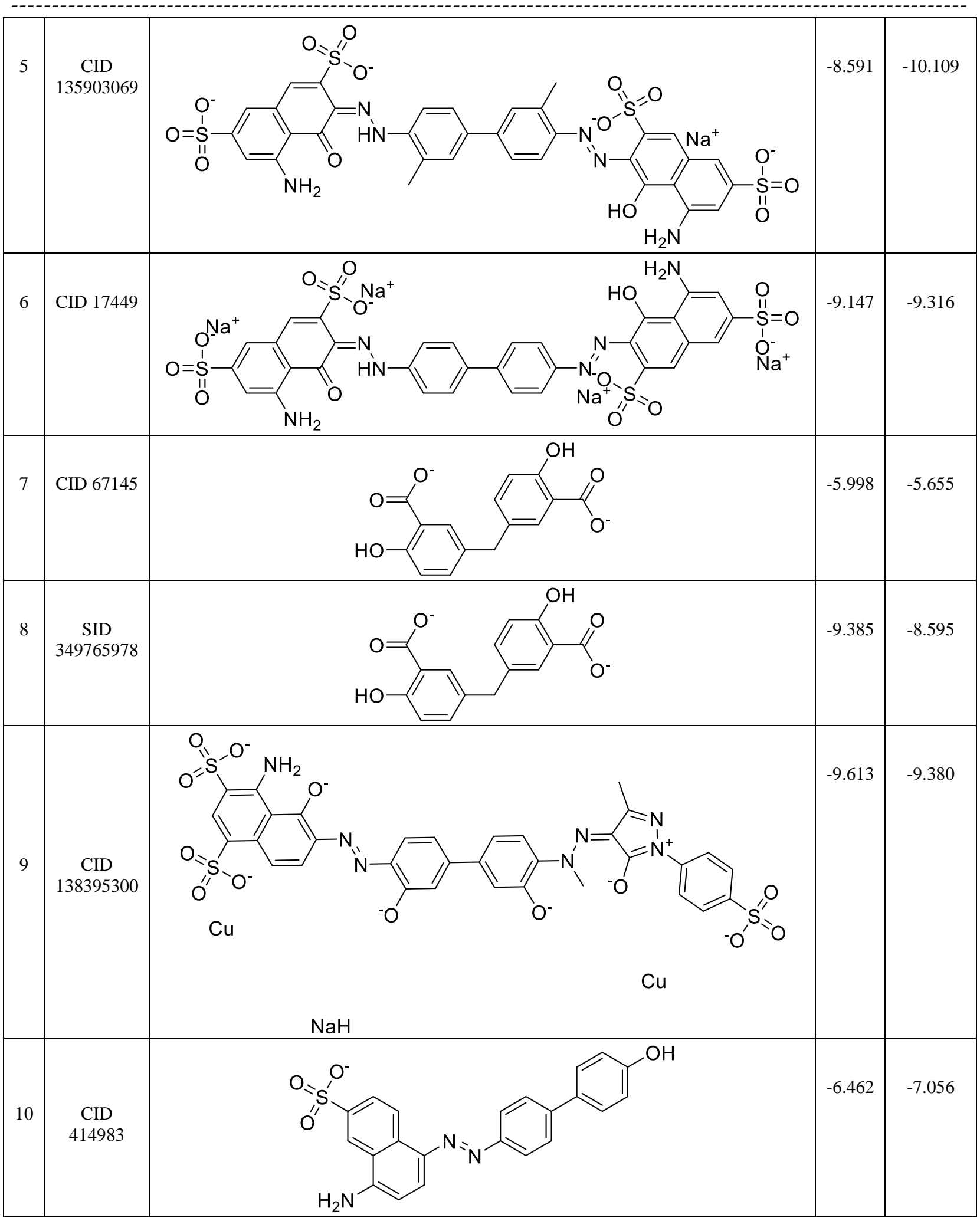


Docking of (PNAI) inhibitor molecules for SARS-COV-2

Table 1.2: Details of ligand and receptor interaction of molecules (1-10) with 6W4H (PNAI) at pH 7.

\begin{tabular}{|c|c|c|c|c|c|c|}
\hline S.no. & $\begin{array}{c}\text { PubChem } \\
\text { code }\end{array}$ & $\begin{array}{c}\text { Total } \\
\text { Binding } \\
\text { Energy } \\
\text { Kcal/mol } \\
\end{array}$ & Ligand & Receptor & Interaction & Distance \\
\hline \multirow[t]{14}{*}{1} & CID 428775 & -8.57129 & $\begin{array}{ll}\mathrm{N} & 14\end{array}$ & O $\quad$ CYS 4294 (B) & H-donor & 3.01 \\
\hline & & & O 6 & ND2 ASN 6996 (A) & H-acceptor & 2.99 \\
\hline & & & O 6 & OG $\quad$ SER $6999(\mathrm{~A})$ & H-acceptor & 3.16 \\
\hline & & & $\begin{array}{ll}\mathrm{O} & 8\end{array}$ & NZ $\quad$ LYS 4296 (B) & H-acceptor & 3.47 \\
\hline & & & $\mathrm{O} 4$ & NZ $\quad$ LYS $6836(\mathrm{~A})$ & ionic & 3.00 \\
\hline & & & O 5 & NZ $\quad$ LYS 6844 (A) & ionic & 2.94 \\
\hline & & & O 5 & NZ $\quad$ LYS 6968 (A) & ionic & 3.53 \\
\hline & & & $\begin{array}{ll}\mathrm{O} & 7\end{array}$ & NZ $\quad$ LYS 6836 (A) & ionic & 2.84 \\
\hline & & & O 7 & NZ $\quad$ LYS 4296 (B) & ionic & 2.94 \\
\hline & & & O 8 & NZ LYS 4296 (B) & ionic & 3.47 \\
\hline & & & 6-ring & MET 6839 (A) & П-H & 4.07 \\
\hline & & & 6-ring & $\mathrm{N} \quad$ MET $6840(\mathrm{~A})$ & П-H & 3.98 \\
\hline & & & 6-ring & NZ $\quad$ LYS 6844 (A) & Pi cation & 4.73 \\
\hline & & & 6-ring & NZ $\quad$ LYS 4296 (B) & pi-cation & 4.53 \\
\hline \multirow[t]{8}{*}{2} & CID 11314 & -8.24403 & O 5 & SD $\quad$ MET $6929(A)$ & H-donor & 2.87 \\
\hline & & & O 6 & SD $\quad$ MET $6929(\mathrm{~A})$ & H-donor & 3.03 \\
\hline & & & $\mathrm{N} 9$ & $\begin{array}{ll}\text { O } & \text { TYR } 6930(\mathrm{~A})\end{array}$ & H-donor & 3.05 \\
\hline & & & O 5 & N CYS 6913 (A) & H-acceptor & 3.09 \\
\hline & & & $\mathrm{O} 8$ & NZ $\quad$ LYS 6968 (A) & H-acceptor & 3.13 \\
\hline & & & 6-ring & CD2 LEU 6898 (A) & П-H & 3.71 \\
\hline & & & 6-ring & ND2 ASN 6996 (A) & П-H & 4.34 \\
\hline & & & 6-ring & ND2 ASN 6996 (A) & П-H & 3.88 \\
\hline \multirow[t]{10}{*}{3} & CID 2259 & -6.28164 & O 1 & ND2 ASN 6996 (A) & H-acceptor & 3.08 \\
\hline & & & O 3 & NZ $\quad$ LYS $6874(\mathrm{~A})$ & H-acceptor & 2.97 \\
\hline & & & $\mathrm{O} 4$ & NZ $\quad$ LYS 6968 (A) & H-acceptor & 3.10 \\
\hline & & & O 5 & $\begin{array}{lll}\text { CA } & \text { SER } 6872(A)\end{array}$ & H-acceptor & 3.31 \\
\hline & & & O 5 & N $\quad$ ASP $6873(A)$ & H-acceptor & 3.14 \\
\hline & & & O 6 & $\mathrm{~N} \quad$ MET $6840(\mathrm{~A})$ & H-acceptor & 3.08 \\
\hline & & & O 7 & ND2 ASN 6996 (A) & H-acceptor & 3.05 \\
\hline & & & O 7 & OG $\quad$ SER $6999(\mathrm{~A})$ & H-acceptor & 2.94 \\
\hline & & & O 9 & N $\quad$ ASN 6841 (A) & H-acceptor & 2.99 \\
\hline & & & O 4 & NZ $\quad$ LYS 6844 (A) & ionic & 3.88 \\
\hline
\end{tabular}


Docking of (PNAI) inhibitor molecules for SARS-COV-2

\begin{tabular}{|c|c|c|c|c|c|c|}
\hline & & & $\mathrm{O} 4$ & NZ $\quad$ LYS 6968 (A) & ionic & 3.10 \\
\hline & & & O 6 & NZ LYS $6874(\mathrm{~A})$ & ionic & 3.78 \\
\hline \multirow[t]{6}{*}{4} & CID 160843 & -7.68205 & $\mathrm{O} 3$ & N $\quad$ CYS 6913 (A) & H-acceptor & 3.09 \\
\hline & & & O 5 & NZ $\quad$ LYS 6874 (A) & H-acceptor & 2.97 \\
\hline & & & O 7 & $\mathrm{~N} \quad$ MET $6840(\mathrm{~A})$ & H-acceptor & 3.16 \\
\hline & & & $\mathrm{O} 8$ & N $\quad$ GLY 6869 (A) & H-acceptor & 3.12 \\
\hline & & & $\mathrm{O} 5$ & NZ LYS $6874(\mathrm{~A})$ & ionic & 2.97 \\
\hline & & & O 7 & NZ $\quad$ LYS $6874(\mathrm{~A})$ & ionic & 3.58 \\
\hline \multirow[t]{11}{*}{5} & $\begin{array}{c}\text { CID } \\
135903069\end{array}$ & -10.1096 & $\begin{array}{ll}\mathrm{O} & 14\end{array}$ & SG $\quad$ CYS $4294(B)$ & H-donor & 3.31 \\
\hline & & & $\begin{array}{ll}\mathrm{O} & 7\end{array}$ & $\begin{array}{lll}\text { NZ } & \text { LYS } & 6968(A)\end{array}$ & H-acceptor & 2.93 \\
\hline & & & O 13 & NZ LYS 6935 (A) & H-acceptor & 2.91 \\
\hline & & & $\begin{array}{ll}\mathrm{O} & 14\end{array}$ & N $\quad$ CYS 4294 (B) & H-acceptor & 3.25 \\
\hline & & & O 15 & $\mathrm{~N} \quad \mathrm{SER} 7000(\mathrm{~A})$ & H-acceptor & 3.13 \\
\hline & & & O 15 & OG $\quad$ SER $7000(\mathrm{~A})$ & H-acceptor & 2.74 \\
\hline & & & O 7 & NZ $\quad$ LYS $6968(\mathrm{~A})$ & ionic & 2.93 \\
\hline & & & $\begin{array}{ll}\mathrm{O} & 10\end{array}$ & NZ $\quad$ LYS 6844 (A) & ionic & 2.96 \\
\hline & & & $\begin{array}{ll}\mathrm{O} & 10\end{array}$ & NZ $\quad$ LYS 6968 (A) & ionic & 3.58 \\
\hline & & & $\begin{array}{ll}\mathrm{O} & 11\end{array}$ & NZ $\quad$ LYS $6874(A)$ & ionic & 3.14 \\
\hline & & & $\begin{array}{ll} & 13\end{array}$ & NZ $\quad$ LYS 6935 (A) & ionic & 2.91 \\
\hline \multirow[t]{12}{*}{6} & CID 17449 & -9.31642 & $\begin{array}{ll}\mathrm{O} & 16\end{array}$ & SG $\quad$ CYS 4294 (B) & H-donor & 3.49 \\
\hline & & & $\begin{array}{ll}\mathrm{O} & 12\end{array}$ & NZ $\quad$ LYS 6968 (A) & H-acceptor & 2.90 \\
\hline & & & O 14 & N $\quad$ SER $7000(A)$ & H-acceptor & 2.91 \\
\hline & & & $\begin{array}{ll}\mathrm{O} & 14\end{array}$ & OG $\quad$ SER $7000(\mathrm{~A})$ & H-acceptor & 2.70 \\
\hline & & & $\begin{array}{ll}\mathrm{O} & 16\end{array}$ & N $\quad$ CYS 4294 (B) & H-acceptor & 3.10 \\
\hline & & & $\begin{array}{ll}\mathrm{O} & 17\end{array}$ & NZ $\quad$ LYS 6935 (A) & H-acceptor & 2.79 \\
\hline & & & O 7 & NZ LYS $6874(\mathrm{~A})$ & ionic & 2.86 \\
\hline & & & O 8 & NZ $\quad$ LYS 6844 (A) & ionic & 2.95 \\
\hline & & & O 8 & NZ LYS 6968 (A) & ionic & 3.69 \\
\hline & & & O 12 & NZ LYS 6968 (A) & ionic & 2.90 \\
\hline & & & $\begin{array}{ll}\mathrm{O} & 17\end{array}$ & NZ $\quad$ LYS 6935 (A) & ionic & 2.79 \\
\hline & & & 6-ring & $\mathrm{N} \quad$ MET $6840(\mathrm{~A})$ & П-H & 3.84 \\
\hline \multirow[t]{4}{*}{7} & CID 67145 & -5.65501 & $\begin{array}{ll} & 1\end{array}$ & NZ LYS 6844 (A) & H-acceptor & 3.05 \\
\hline & & & $\mathrm{O} 5$ & NZ $\quad$ LYS $6968(\mathrm{~A})$ & H-acceptor & 3.27 \\
\hline & & & O 7 & NZ LYS 6968 (A) & H-acceptor & 2.96 \\
\hline & & & O 8 & $\begin{array}{lll}\text { OH } & \text { TYR } 6930(A)\end{array}$ & H-acceptor & 3.06 \\
\hline
\end{tabular}


Docking of (PNAI) inhibitor molecules for SARS-COV-2

\begin{tabular}{|c|c|c|c|c|c|c|}
\hline & & & O 5 & NZ $\quad$ LYS 6844 (A) & ionic & 2.96 \\
\hline & & & O 5 & NZ LYS 6968 (A) & ionic & 3.27 \\
\hline & & & O 7 & NZ LYS $6968(A)$ & ionic & 2.96 \\
\hline & & & 6-ring & NZ LYS 6935 (A) & pi-cation & 3.94 \\
\hline \multirow[t]{3}{*}{8} & $\begin{array}{c}\text { SID } \\
349765978\end{array}$ & -8.59571 & $\mathrm{O} \quad 2$ & NZ LYS 6968 (A) & H-acceptor & 2.98 \\
\hline & & & O 7 & N $\quad$ CYS 6913 (A) & H-acceptor & 3.17 \\
\hline & & & O 7 & SG CYS $6913(\mathrm{~A})$ & H-acceptor & 3.32 \\
\hline \multirow[t]{10}{*}{9} & $\begin{array}{c}\text { CID } \\
138395300\end{array}$ & -9.38083 & O 8 & NZ $\quad$ LYS $6836(A)$ & $\mathrm{H}$-acceptor & 2.92 \\
\hline & & & O 15 & NZ $\quad$ LYS 6935 (A) & $\mathrm{H}$-acceptor & 3.47 \\
\hline & & & O 16 & OG $\quad$ SER $7000(\mathrm{~A})$ & H-acceptor & 2.94 \\
\hline & & & $\begin{array}{ll} \\
\end{array}$ & NZ LYS $6836(\mathrm{~A})$ & ionic & 2.92 \\
\hline & & & O 8 & NZ LYS 4296 (B) & ionic & 3.87 \\
\hline & & & O 10 & NZ LYS $6836(A)$ & ionic & 3.52 \\
\hline & & & $\begin{array}{ll}\mathrm{O} & 11\end{array}$ & NZ $\quad$ LYS $6844(A)$ & ionic & 3.76 \\
\hline & & & $\begin{array}{ll}\mathrm{O} & 13\end{array}$ & NZ $\quad$ LYS 6874 (A) & ionic & 2.80 \\
\hline & & & O 15 & NZ LYS 6935 (A) & ionic & 3.47 \\
\hline & & & 5-ring & NZ $\quad$ LYS $6968(A)$ & pi-cation & 4.26 \\
\hline \multirow[t]{3}{*}{10} & CID 414983 & -7.05687 & O 2 & O $\quad$ GLY 6871 (A) & H-donor & 3.12 \\
\hline & & & $\begin{array}{ll}\mathrm{O} & 4\end{array}$ & ND2 ASN 6841 (A) & H-acceptor & 2.99 \\
\hline & & & 6-ring & CD2 PHE $6947(\mathrm{~A})$ & П-H & 3.95 \\
\hline
\end{tabular}


Docking of (PNAI) inhibitor molecules for SARS-COV-2

Table 1.2: Details of ligand and receptor interaction of molecules (1-10) with 6W4H (PNAI) at $\mathrm{pH} 5$.

\begin{tabular}{|c|c|c|c|c|c|c|}
\hline S.no. & $\begin{array}{c}\text { PubChem } \\
\text { code }\end{array}$ & $\begin{array}{c}\text { Total Binding Energy } \\
\text { Kcal/mol }\end{array}$ & Ligand & Receptor & Interaction & Distance \\
\hline \multirow[t]{15}{*}{1} & $\begin{array}{c}\text { CID } \\
428775\end{array}$ & -8.57129 & $\begin{array}{ll}\mathrm{N} & 13\end{array}$ & O $\quad$ CYS 4294 (B) & H-donor & 3.04 \\
\hline & & & $\begin{array}{ll}\mathrm{N} & 14\end{array}$ & OG $\quad$ SER $6831(\mathrm{~A})$ & H-donor & 3.22 \\
\hline & & & O 7 & NZ $\quad$ LYS $6968(A)$ & $\mathrm{H}$-acceptor & 3.45 \\
\hline & & & $\begin{array}{ll}\mathrm{O} & 8\end{array}$ & ND2 ASN $6996(A)$ & H-acceptor & 3.03 \\
\hline & & & O 8 & OG $\quad$ SER $6999(\mathrm{~A})$ & $\mathrm{H}$-acceptor & 3.10 \\
\hline & & & O 3 & NZ $\quad$ LYS $6836(\mathrm{~A})$ & ionic & 2.95 \\
\hline & & & O 5 & NZ $\quad$ LYS 6836 (A) & ionic & 2.84 \\
\hline & & & O 5 & $\begin{array}{lll}\text { NZ } & \text { LYS } & 4296 \text { (B) }\end{array}$ & ionic & 2.97 \\
\hline & & & O 6 & NZ LYS 4296 (B) & ionic & 3.63 \\
\hline & & & O 7 & NZ $\quad$ LYS 6844 (A) & ionic & 2.99 \\
\hline & & & O 7 & NZ $\quad$ LYS $6968(\mathrm{~A})$ & ionic & 3.45 \\
\hline & & & 6-ring & CA $\quad$ MET 6839 (A) & П-H & 4.04 \\
\hline & & & 6-ring & $\mathrm{N} \quad \mathrm{MET} 6840(\mathrm{~A})$ & П-H & 3.90 \\
\hline & & & 6-ring & $\begin{array}{lll}\text { NZ } & \text { LYS } & 6844(\mathrm{~A})\end{array}$ & pi-cation & 4.65 \\
\hline & & & 6-ring & NZ LYS 4296 (B) & pi-cation & 4.58 \\
\hline \multirow[t]{10}{*}{2} & CID 11314 & -8.24403 & $\mathrm{~N} 9$ & O $\quad$ CYS 4294 (B) & H-donor & 3.35 \\
\hline & & & O 3 & NZ LYS 4296 (B) & H-acceptor & 3.54 \\
\hline & & & $\mathrm{O} 4$ & ND2 ASN 6996 (A) & H-acceptor & 2.93 \\
\hline & & & $\mathrm{O} 4$ & $\begin{array}{ll}\text { OG } & \text { SER } 6999(\mathrm{~A})\end{array}$ & H-acceptor & 3.09 \\
\hline & & & O 8 & $\begin{array}{lll}\text { NZ } & \text { LYS } & 6844(\mathrm{~A})\end{array}$ & H-acceptor & 3.08 \\
\hline & & & O 3 & NZ LYS 4296 (B) & ionic & 3.54 \\
\hline & & & O 8 & NZ $\quad$ LYS $6844(A)$ & ionic & 3.08 \\
\hline & & & 6-ring & CA $\quad$ MET $6839(\mathrm{~A})$ & П-H & 3.91 \\
\hline & & & 6-ring & $\mathrm{N} \quad$ MET $6840(\mathrm{~A})$ & П-H & 4.65 \\
\hline & & & 6-ring & N $\quad$ MET $6840(\mathrm{~A})$ & П-H & 4.11 \\
\hline \multirow[t]{3}{*}{3} & CID 2259 & -6.28164 & $\mathrm{O} 3$ & SG CYS $6913(\mathrm{~A})$ & H-acceptor & 3.51 \\
\hline & & & O 9 & $\mathrm{~N} \quad \mathrm{CYS} 6913$ (A) & H-acceptor & 2.90 \\
\hline & & & 6-ring & CA $\quad$ ASP $6931(\mathrm{~A})$ & П-H & 4.35 \\
\hline 4 & $\begin{array}{c}\text { CID } \\
160843 \\
\end{array}$ & -7.68205 & O 3 & N CYS 6913 (A) & H-acceptor & 3.15 \\
\hline
\end{tabular}


Docking of (PNAI) inhibitor molecules for SARS-COV-2

\begin{tabular}{|c|c|c|c|c|c|c|}
\hline & & & $\begin{array}{ll}\mathrm{O} & 8\end{array}$ & $\begin{array}{lll}\mathrm{N} & \text { GLY } & 6869 \text { (A) }\end{array}$ & H-acceptor & 3.09 \\
\hline & & & 6-ring & $\mathrm{N} \quad$ ASN $6841(\mathrm{~A})$ & П-H & 4.65 \\
\hline \multirow[t]{12}{*}{5} & $\begin{array}{c}\text { CID } \\
135903069\end{array}$ & -10.1096 & $\mathrm{~N} 2$ & OD1 ASN 4293 (B) & H-donor & 2.88 \\
\hline & & & O 5 & NZ $\quad$ LYS 6874 (A) & H-acceptor & 3.23 \\
\hline & & & $\mathrm{O}$ & $\begin{array}{lll}\text { NZ } & \text { LYS } & 6844(\mathrm{~A})\end{array}$ & H-acceptor & 2.92 \\
\hline & & & $\begin{array}{ll}\mathrm{O} & 9\end{array}$ & ND2 ASN 6996 (A) & H-acceptor & 3.07 \\
\hline & & & $\begin{array}{ll}\mathrm{O} & 12\end{array}$ & $\begin{array}{lll}\text { NZ } & \text { LYS } & 6968(\mathrm{~A})\end{array}$ & H-acceptor & 3.14 \\
\hline & & & $\begin{array}{ll}\mathrm{O} & 15\end{array}$ & $\begin{array}{lll}\text { OH } & \text { TYR } & 6930(\mathrm{~A})\end{array}$ & H-acceptor & 3.06 \\
\hline & & & $\begin{array}{ll}\mathrm{O} & 18\end{array}$ & $\begin{array}{lll}\mathrm{N} & \text { HIS } & 6972(\mathrm{~A})\end{array}$ & H-acceptor & 3.19 \\
\hline & & & O 19 & CD2 HIS $6972(\mathrm{~A})$ & H-acceptor & 3.43 \\
\hline & & & O 9 & NZ $\quad$ LYS 6844 (A) & ionic & 2.92 \\
\hline & & & $\begin{array}{ll}\mathrm{O} & 12\end{array}$ & NZ $\quad$ LYS 6968 (A) & ionic & 3.14 \\
\hline & & & $\begin{array}{ll}\text { O } & 19\end{array}$ & NZ $\quad$ LYS 6935 (A) & ionic & 3.65 \\
\hline & & & 6-ring & $\begin{array}{lll}\mathrm{N} & \text { SER } 7000(\mathrm{~A})\end{array}$ & П-H & 4.29 \\
\hline \multirow[t]{14}{*}{6} & CID 17449 & -9.31642 & O 18 & SG CYS $4294(B)$ & H-donor & 3.29 \\
\hline & & & $\mathrm{N} \quad 23$ & $\begin{array}{lll}\text { O } & \text { GLY } & 6837 \text { (A) }\end{array}$ & H-donor & 3.02 \\
\hline & & & $\mathrm{O} 8$ & NZ $\quad$ LYS 6844 (A) & H-acceptor & 3.01 \\
\hline & & & $\begin{array}{ll} & 10\end{array}$ & NZ $\quad$ LYS 6844 (A) & H-acceptor & 2.96 \\
\hline & & & $\begin{array}{ll}\mathrm{O} & 14\end{array}$ & $\begin{array}{lll}\mathrm{N} & \text { SER } 7000(\mathrm{~A})\end{array}$ & H-acceptor & 3.37 \\
\hline & & & $\begin{array}{ll}\mathrm{O} & 16\end{array}$ & NZ $\quad$ LYS 6935 (A) & H-acceptor & 2.82 \\
\hline & & & $\begin{array}{ll}\mathrm{O} & 17\end{array}$ & $\begin{array}{ll}\mathrm{N} & \text { SER } 7000(\mathrm{~A})\end{array}$ & H-acceptor & 3.33 \\
\hline & & & $\begin{array}{ll}\mathrm{O} & 17\end{array}$ & $\begin{array}{ll}\text { OG } & \text { SER } 7000(\mathrm{~A})\end{array}$ & H-acceptor & 2.82 \\
\hline & & & O 8 & NZ $\quad$ LYS 6844 (A) & ionic & 3.01 \\
\hline & & & O 9 & NZ $\quad$ LYS 6874 (A) & ionic & 2.92 \\
\hline & & & $\begin{array}{ll}\mathrm{O} & 10\end{array}$ & NZ $\quad$ LYS 6844 (A) & ionic & 2.96 \\
\hline & & & $\begin{array}{ll}\mathrm{O} & 16\end{array}$ & NZ $\quad$ LYS 6935 (A) & ionic & 2.82 \\
\hline & & & 6-ring & $\begin{array}{lll}\text { NZ } & \text { LYS } & 6874(\mathrm{~A})\end{array}$ & pi-cation & 4.67 \\
\hline & & & 6-ring & ND2 ASN $6996(A)$ & П-H & 4.22 \\
\hline \multirow[t]{2}{*}{7} & CID 67145 & -5.65501 & O 5 & $\begin{array}{lll}\mathrm{N} & \mathrm{CYS} & 6913(\mathrm{~A})\end{array}$ & H-acceptor & 3.14 \\
\hline & & & 6-ring & $\begin{array}{lll}\mathrm{N} & \text { TYR } 6930(\mathrm{~A})\end{array}$ & П-H & 4.61 \\
\hline
\end{tabular}


Docking of (PNAI) inhibitor molecules for SARS-COV-2

\begin{tabular}{|c|c|c|c|c|c|c|}
\hline 8 & $\begin{array}{c}\text { SID } \\
349765978\end{array}$ & -8.59571 & $\begin{array}{ll}\mathrm{N} & 11\end{array}$ & OD1 $\quad$ ASP 6897 (A) & H-donor & 3.33 \\
\hline & & & $\begin{array}{ll}\mathrm{O} & 1\end{array}$ & $\begin{array}{ll}\mathrm{N} & \text { TYR } 6930(\mathrm{~A})\end{array}$ & H-acceptor & 2.93 \\
\hline & & & $\mathrm{O} \quad 2$ & NZ $\quad$ LYS 6968 (A) & H-acceptor & 2.91 \\
\hline & & & $\begin{array}{ll}O & 6\end{array}$ & NZ $\quad$ LYS 6844 (A) & H-acceptor & 3.01 \\
\hline & & & O 8 & N $\quad$ GLY 6869 (A) & H-acceptor & 3.02 \\
\hline & & & O 6 & NZ $\quad$ LYS 6844 (A) & ionic & 3.01 \\
\hline & & & 6-ring & ND2 ASN $6996(A)$ & П-H & 4.79 \\
\hline \multirow[t]{16}{*}{9} & $\begin{array}{c}\text { CID } \\
138395300 \\
\end{array}$ & -9.38083 & $\mathrm{O} 4$ & ND2 ASN 6996 (A) & H-acceptor & 2.92 \\
\hline & & & $\mathrm{O} 4$ & OG SER $6999(\mathrm{~A})$ & $\mathrm{H}$-acceptor & 3.28 \\
\hline & & & $\mathrm{O}$ & $\mathrm{N} \quad$ GLY 6829 (A) & $\mathrm{H}$-acceptor & 3.31 \\
\hline & & & $\mathrm{O}$ & NZ LYS 6935 (A) & H-acceptor & 2.77 \\
\hline & & & $\mathrm{O}$ & NZ LYS 6935 (A) & H-acceptor & 3.50 \\
\hline & & & O 10 & OG SER $7000(\mathrm{~A})$ & $\mathrm{H}$-acceptor & 3.05 \\
\hline & & & O 12 & CA SER $6872(A)$ & $\mathrm{H}$-acceptor & 3.17 \\
\hline & & & 12 & $\mathrm{~N} \quad$ ASP $6873(\mathrm{~A})$ & H-acceptor & 3.16 \\
\hline & & & O 13 & NZ LYS 6844 (A) & $\mathrm{H}$-acceptor & 2.75 \\
\hline & & & 14 & CA ASP $6912(\mathrm{~A})$ & $\mathrm{H}$-acceptor & 3.34 \\
\hline & & & 15 & SG CYS $6913(\mathrm{~A})$ & H-acceptor & 3.23 \\
\hline & & & $\mathrm{O}$ & NZ LYS 6935 (A) & ionic & 2.77 \\
\hline & & & $\begin{array}{ll} & 9 \\
\end{array}$ & NZ LYS 6935 (A) & ionic & 3.50 \\
\hline & & & $\begin{array}{ll}\mathrm{O} & 13\end{array}$ & NZ LYS 6844 (A) & ionic & 2.75 \\
\hline & & & $\begin{array}{ll}\mathrm{N} & 18\end{array}$ & OD1 $\quad$ ASP $6897(\mathrm{~A})$ & ionic & 3.28 \\
\hline & & & $\mathrm{N} \quad 18$ & OD2 ASP $6897(\mathrm{~A})$ & ionic & 3.38 \\
\hline \multirow[t]{7}{*}{10} & $\begin{array}{c}\text { CID } \\
414983 \\
\end{array}$ & -7.05687 & O 3 & NZ LYS 6968 (A) & $\mathrm{H}$-acceptor & 2.94 \\
\hline & & & $\begin{array}{ll} \\
\end{array}$ & NZ $\quad$ LYS $6844(\mathrm{~A})$ & $\mathrm{H}$-acceptor & 2.92 \\
\hline & & & O 3 & NZ LYS 6844 (A) & ionic & 3.80 \\
\hline & & & O 3 & NZ LYS 6968 (A) & ionic & 2.94 \\
\hline & & & $\mathrm{O} 4$ & NZ $\quad$ LYS $6844(A)$ & ionic & 2.92 \\
\hline & & & 6-ring & CB LEU $6898(\mathrm{~A})$ & П-H & 3.57 \\
\hline & & & 6-ring & $\begin{array}{lll}\mathrm{N} & \text { TYR } 6930(\mathrm{~A})\end{array}$ & П-H & 4.91 \\
\hline
\end{tabular}




\section{Docking of (PNAI) inhibitor molecules for SARS-COV-2}

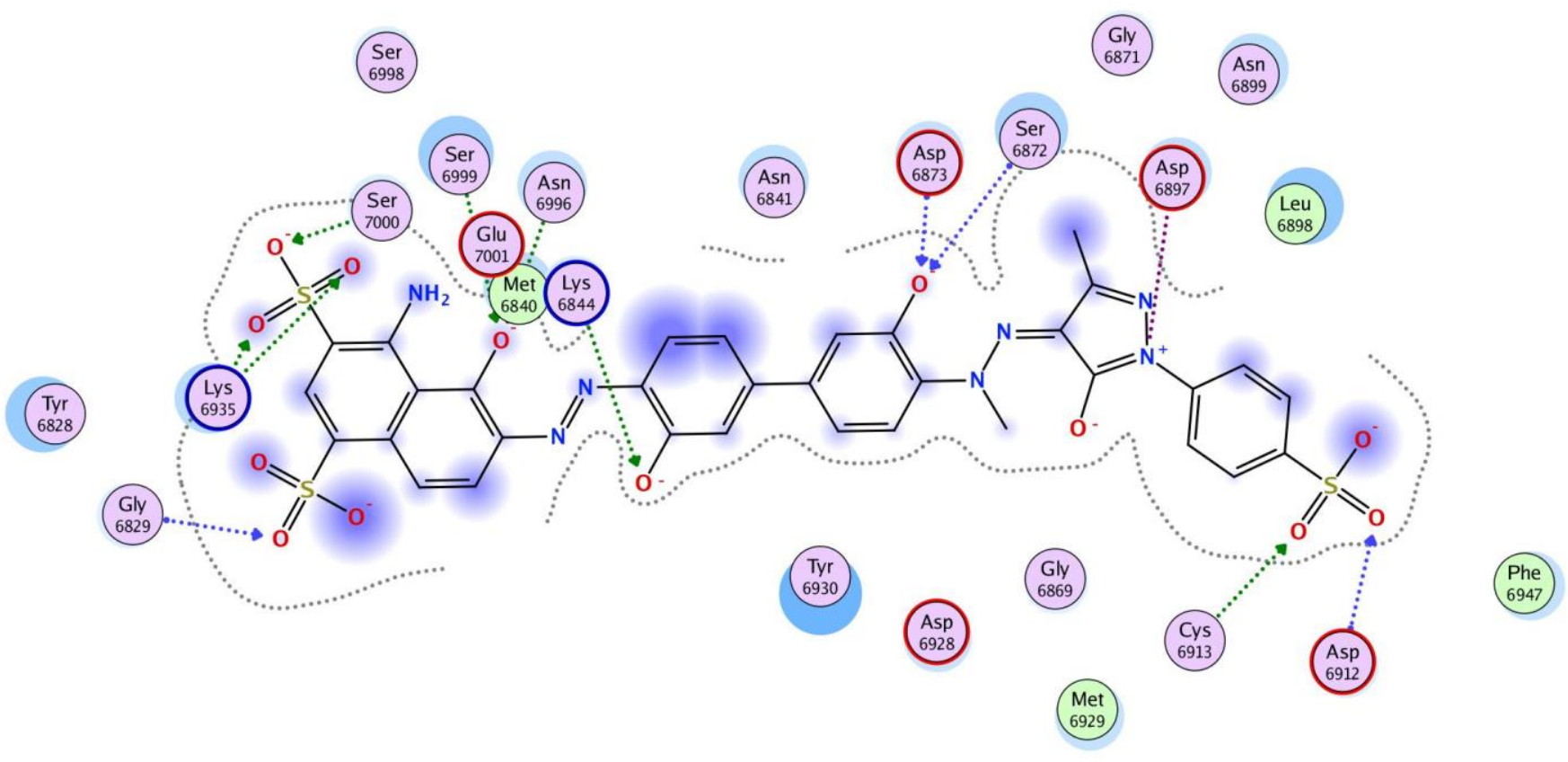

Figure-1. Binding interactions of (9) with 6W4H PNAI at pH 5.

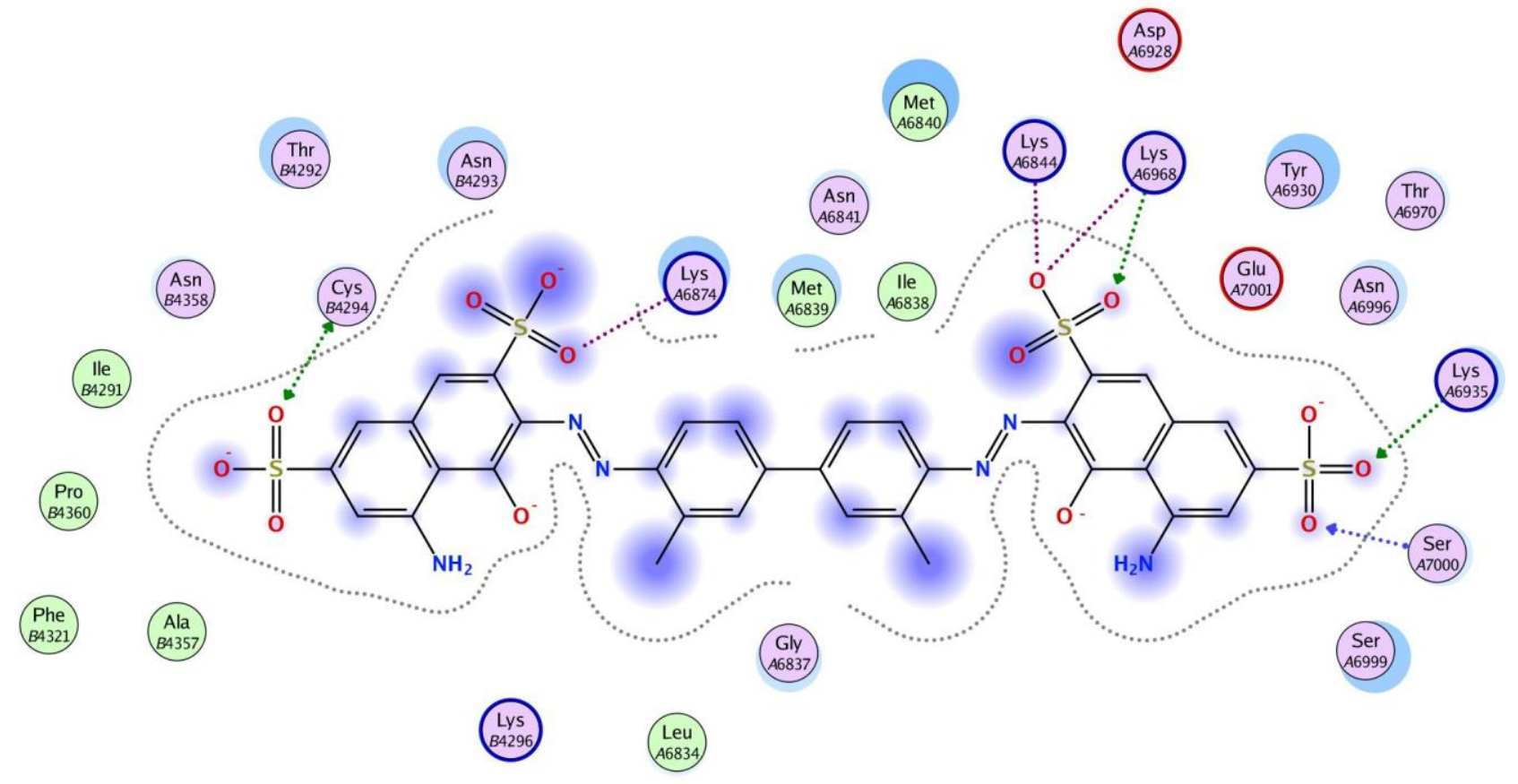

Reference

Figure-1. Binding interactions of (5) with 6W4H PNAI at pH 7. 
Docking of (PNAI) inhibitor molecules for SARS-COV-2

1. Hui, D. S.; I Azhar, E.; Madani, T. A.; Ntoumi, F.; Kock, R.; Dar, O.; Ippolito, G.; Mchugh, T. D.; Memish, Z. A.; Drosten, C.; et al. The Continuing 2019-NCoV Epidemic Threat of Novel Coronaviruses to Global Health - The Latest 2019 Novel Coronavirus Outbreak in Wuhan, China. International Journal of Infectious Diseases 2020, 91, 264-266. https://doi.org/10.1016/j.ijid.2020.01.009.

2. Han, D. P.; Penn-Nicholson, A.; Cho, M. W. Identification of Critical Determinants on ACE2 for SARS-CoV Entry and Development of a Potent Entry Inhibitor. Virology 2006, 350 (1), 15-25. https://doi.org/10.1016/j.virol.2006.01.029.

3. Li, G.; De Clercq, E. Therapeutic Options for the 2019 Novel Coronavirus (2019-NCoV). Nat Rev Drug Discov 2020, d41573-02000016-0. https://doi.org/10.1038/d41573020-00016-0.

4. Cameron, C. E.; Castro, C. The Mechanism of Action of Ribavirin: Lethal Mutagenesis of RNA Virus Genomes Mediated by the Viral RNA-Dependent RNA Polymerase: Current Opinion in Infectious Diseases 2001, $14 \quad$ (6), 757-764. https://doi.org/10.1097/00001432200112000-00015.

5. Chu, C. M. Role of Lopinavir/Ritonavir in the Treatment of SARS: Initial Virological and Clinical Findings. Thorax 2004, 59 (3), 252-256.

https://doi.org/10.1136/thorax.2003.012658.

6. Lu, I.-L.; Mahindroo, N.; Liang, P.-H.; Peng, Y.-H.; Kuo, C.-J.; Tsai, K.-C.; Hsieh, H.-P.; Chao, Y.-S.; Wu, S.-Y. Structure-Based Drug Design and Structural Biology Study of Novel Nonpeptide Inhibitors of Severe Acute Respiratory Syndrome Coronavirus Main Protease. J. Med. Chem. 2006, 49 (17), 51545161. https://doi.org/10.1021/jm060207o.

7. Blanchard, J. E.; Elowe, N. H.; Huitema, C.; Fortin, P. D.; Cechetto, J. D.; Eltis, L. D.; Brown, E. D. High-Throughput Screening
Identifies Inhibitors of the SARS Coronavirus Main Proteinase. Chemistry \& Biology 2004, 11 (10), 1445-1453. https://doi.org/10.1016/j.chembiol.2004.08.0 11.

8. Liu, X.; Zhang, B.; Jin, Z.; Yang, H.; Rao, Z. The Crytal Structure of 2019-NCoV Main Protease in Complex with an Inhibitor N3. $P D B$ 2020. https://doi.org/10.2210/pdb6lu7/pdb.

9. Paasche, A.; Zipper, A.; Schäfer, S.; Ziebuhr, J.; Schirmeister, T.; Engels, B. Evidence for Substrate Binding-Induced Zwitterion Formation in the Catalytic Cys-His Dyad of the SARS-CoV Main Protease. Biochemistry 2014, 53 (37), 5930-5946. https://doi.org/10.1021/bi400604t.

10. Lee, H.; Mittal, A.; Patel, K.; Gatuz, J. L.; Truong, L.; Torres, J.; Mulhearn, D. C.; Johnson, M. E. Identification of Novel Drug Scaffolds for Inhibition of SARS-CoV 3Chymotrypsin-like Protease Using Virtual and High-Throughput Screenings. Bioorg. Med. Chem. 2014, 22 (1), 167-177. https://doi.org/10.1016/j.bmc.2013.11.041.

11. Ghosh, A. K.; Xi, K.; Johnson, M. E.; Baker, S. C.; Mesecar, A. D. Progress in Anti-SARS Coronavirus Chemistry, Biology and Chemotherapy. In Annual Reports in Med Chem. (2006), 41, 183-196. https://doi.org/10.1016/S00657743(06)41011-3.

12. Tuley, A.; Fast, W. The Taxonomy of Covalent Inhibitors. Biochemistry 2018, 57 (24), 3326-3337. https://doi.org/10.1021/acs.biochem.8b0031 5 .

13. Turk, B. Targeting Proteases: Successes, Failures and Future Prospects. Nat Rev Drug Discov 2006, 5 (9), 785-799. https://doi.org/10.1038/nrd2092.

14. Ghosh, A. K.; Gong, G.; Grum-Tokars, V.; Mulhearn, D. C.; Baker, S. C.; Coughlin, M.; Prabhakar, B. S.; Sleeman, K.; Johnson, M. E.; Mesecar, A. D. Design, Synthesis and 


\section{Docking of (PNAI) inhibitor molecules for SARS-COV-2}

Antiviral Efficacy of a Series of Potent Chloropyridyl Ester-Derived SARS-CoV 3CLpro Inhibitors. Bioorg. Med. Chem. Lett. 2008, $18 \quad$ (20), 5684-5688. https://doi.org/10.1016/j.bmcl.2008.08.082.

15. Li, Y.; Zhang, J.; Wang, N.; Li, H.; Shi, Y.; Guo, G.; Liu, K.; Zeng, H.; Zou, Q. Therapeutic Drugs Targeting 2019-NCoV Main Protease by High-Throughput Screening. BioRxiv. 2020, 2020.01.28.922922. https://doi.org/10.1101/2020.01.28.922922.

16. Xu, Z.; Peng, C.; Shi, Y.; Zhu, Z.; Mu, K.; Wang, X.; Zhu, W. Nelfinavir Was Predicted to $\mathrm{Be}$ a Potential Inhibitor of 2019-NCov Main Protease by an Integrative Approach Combining Homology Modelling, Molecular Docking and Binding Free Energy Calculation. BioRxiv. 2020, 2020.01.27.921627. https://doi.org/10.1101/2020.01.27.921627.

17. Liu, X.; Wang, X.-J. Potential Inhibitors for 2019-NCoV Coronavirus M Protease from Clinically Approved Medicines. bioRxiv 2020, 2020.01.29.924100. https://doi.org/10.1101/2020.01.29.924100.

18. Minasov, G.; Shuvalova, L.; Rosas-Lemus, M.; Kiryukhina, O.; Wiersum, G.; Godzik, A.; Jaroszewski, L.; Stogios, P.J.; Skarina, T.; Satchell, K.J.F. 1.80 Angstrom Resolution Crystal Structure of NSP16 NSP10 Complex from SARS-CoV-2. http://www.rcsb.org/structure/6W4H

19. Decroly, E.; Claire D.; François F.; Mickael B.; Bruno C.; Isabelle I.; Laure G. Crystal Structure and Functional Analysis of the SARS-Coronavirus RNA Cap 2'-OMethyltransferase nsp10/nsp16 Complex. Plos Pathog 2011. https://doi:10.1371/journal.ppat.1002059.

20. O'Boyle, N.M. Open Babel: An open chemical toolbox. J Cheminform. 2011, 3, 33. 7;3:33. https://doi: 10.1186/1758-2946-3-33.
21. Berman. H.; The Protein Data Bank: a historical perspective. Acta Cryst. A. 2008, 47, 88-95.

22. Alcantara, J.; Ortiz, K.; Bhardwaj, S.; Palaninathan, M.; Frieman, R. Baric, and C. Kao. Small molecule inhibitors of the SARSCoV Nsp15 endoribonuclease. Virus Adapt. Treat. 2010, 125-133. https://doi.org/10.2147/VAAT.S12733.

23. Liu, C.; Qiongqiong Z.; Yingzhu L.; Linda V. G.; Steve P. W. Research and Development on Therapeutic Agents and Vaccines for COVID-19 and Related Human Coronavirus Diseases." ACS Cent. Sci. https://doi.org/10.1021/acscentsci.0c00272.

24. He, R.; Anton A.; Maya T.A.; Jingxin C.; Todd C.; Elsie G.; Yvon D.; Jody B.; Michael D.; Xuguang L. Potent and selective inhibition of SARS coronavirus replication by aurintricarboxylic acid. Biochem Bioph Res Co. 2004, 32: 1199-1203. https://doi.org/10.1016/j.bbrc.2004.06.076.

25. Liu, H.; Maruyama, H.; Masuda, T.; Honda, A.; Arai F. The Influence of Virus Infection on the Extracellular pH of the Host Cell Detected on Cell Membrane. Front Microbiol. 2016, 17(7), 1127. https://doi: 10.3389/fmicb.2016.01127. 